\title{
Joint Train Localization and Track Identification based on Earth Magnetic Field Distortions
}

\author{
Benjamin Siebler*, Oliver Heirich*, Stephan Sand*, Uwe D. Hanebeck ${ }^{\dagger}$ \\ * German Aerospace Center (DLR), Institute of Communications and Navigation \\ ${ }^{\dagger}$ Karlsruhe Institute of Technology (KIT), Intelligent Sensor-Actuator-Systems Laboratory \\ \{benjamin.siebler, stephan.sand\}@dlr.de, uwe.hanebeck@kit.edu
}

\begin{abstract}
In this paper a train localization method is proposed that uses local variations of the earth magnetic field to determine the topological position of a train in a track network. The approach requires a magnetometer triad, an accelerometer, and a map of the magnetic field along the railway tracks. The estimated topological position comprises the along-track position that defines the position of the train within a certain track and the track ID that specifies the track the train is driving on. The along-track position is estimated by a recursive Bayesian filter and the track ID is found from a hypothesis test. In particular the use of multiple particle filter, each estimating the position on different track hypothesis, is proposed. Whenever the estimated train position crosses a switch, a particle filter for each possible track is created. With the position estimates of the different filters, the likelihood for each track hypothesis is calculated from the measured magnetic field and the expected magnetic field in the map. A comparison of the likelihoods is subsequently used to decide which track is the most likely. After a decision for a track is made, the unnecessary filters are deleted. The feasibility of the proposed localization method is evaluated with measurement data recorded on a regional train. In the evaluation, the localization method was running in real time and overall an RMSE below five meter could be achieved and all tracks were correctly identified.
\end{abstract}

\section{INTRODUCTION}

The lack of automation in train traffic leads to large safety distances between consecutives trains and a resulting low density of trains in the track network. Furthermore, current railway systems are not capable to handle flexible and demand driven schedules. This limits the amount of trains on the same track and therefore the passenger and cargo capacity. Especially in urban areas, where the number of passengers constantly grows, this has become a problem that is not easily solved by building new track infrastructure due to substantial costs and limited space. Therefore, automation is one of the best options to increase the traffic density and to make a more flexible use of existing track networks. To enable automation in the future, accurate and continuously available train localization is crucial. In particular, the topological position of each train in the track network is required. The topological position has two components: the along-track position that defines the position of the train within a certain track and the track number that specifies the track the train is driving on. An estimate of the topological position requires some sort of map containing information about the topology of the track network. If the map contains the track geometry in a global coordinate system, e.g., the geocentric earth-centered earthfixed system (ECEF), the topological position can be obtained easily with global navigation satellite system (GNSS) signals by map matching. While in most scenarios, GNSS is sufficient for automated train operation, there are also environments that have a negative effect on the GNSS signals. In tunnels or below roofs in train stations the signals are blocked and in urban areas it is expected that at least severe multipath is encountered. In addition, due to the low signal power at the receiver, GNSS is vulnerable to jamming. Therefore, the detection, the handling and the investigation of possible errors is an important research topic. A survey on GNSS in the railway environment can be found in [1].

Due to the shortcomings of GNSS, we propose to use the magnetic field as an independent source of position information for areas where GNSS signals are degraded or blocked. The use of the magnetic field for localization is based on the observation that ferromagnetic infrastructure components in the vicinity of the track introduces distortions in the earth magnetic field that are persistent over time and characteristic for a certain part of the track. Magnetic field distortions or variations for localization were first considered for indoor localization [2], [3], [4] and have been applied to road vehicles [5] and airplanes [6].

Utilizing magnetic field variations requires a map of the magnetic field along the railway track. The map relates each topological position to the corresponding magnetic field value. For train localization, the measurements of a magnetometer mounted on the train are continuously compared with the map to identify the most likely position. One difficulty in the position estimation is the nonlinear relation between the magnetic field, represented by the map, and the train position. The estimation of the train position therefore requires nonlinear estimators. A simple extended Kalman filter would not work because even position errors of a few meters lead to a wrong linearization of the measurement model. In our prior work [7], we therefore proposed a particle filter that is capable of handling the nonlinearities and we showed that the estimation of the train position only with a magnetometer and motion model is possible with a root mean square error (RMSE) below four meters. Thus far, the correct track ID was assumed to be known in advance. This assumption is not unrealistic in practice, but implies prior knowledge about the whole series of tracks the train traverses. Being able to estimate the track number jointly with the along-track position relaxes this requirement and allows a more flexible use of the magnetic field based localization system. When the series of tracks is already known, the proposed method can serve as detector for faulty switch directions. 

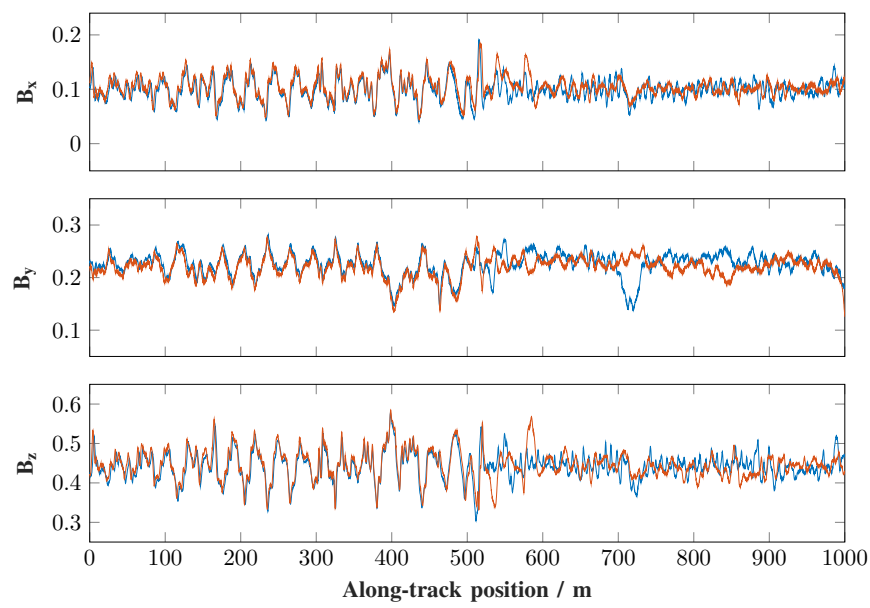

Fig. 1. Magnetic vector field along a $1000 \mathrm{~m}$ long track segment measured during two different runs. Until $500 \mathrm{~m}$, the train was running on the same track during both runs and then a switch was passed and different tracks are traversed. The red and the blue lines show the measurements for the first and second run.

\section{Magnetic Field In The RAilWAy ENVIRONMENT}

The proposed localization system is based on local variations in the earth magnetic field. The variations in the magnetic field are caused by ferromagnetic material that influences the earth magnetic field. In the railway environment, ferromagnetic material is found in the track, the poles holding the overhead line, but also in the substructure of the track and steel reinforced concrete in sound barriers and platforms. Overall, this causes position specific variations that are persistent in time. An example of such variations is shown in Fig. 1. The figure shows the measured components of the magnetic vector field on a $1000 \mathrm{~m}$ long track of the Bayerische Regiobahn (BRB), a local train operator in Augsburg. During the measurements, the $\mathrm{x}$ - and $\mathrm{y}$-axis of the magnetometer were measuring the field in the horizontal plane and the $\mathrm{z}$ axis in the down direction. The $\mathrm{x}$-axis was facing into the driving direction and the y-axis to the right. The values of the magnetic field are normalized by the magnetometer inside the Xsens MTi-G-700 inertial measurement unit (IMU) used during the measurements. The data sheet does not precisely specify the value of the normalization constant but states that it is $\approx 40 \mu \mathrm{T}$. Since the main interest here is the magnetic field and its suitability for localization, not the exact magnetic field strength, this is not an issue.

In Fig. 1, the train was driving on the same track during both runs until $500 \mathrm{~m}$ and than a switch was passed. Before the switch, the magnetic field is very similar for both runs and it can be seen that the value of the magnetic field varies over the along-track position. After the switch is passed, the magnetic field start to differ for the different runs. Fig.1 shows that in principle the magnetic field contains information about the along-track position as well as the track ID and therefore looks promising in respect to the estimation of the topological position. Besides the position specific and time-invariant variations, the magnetometer also measures a magnetic field caused by the current in the overhead line.
The current, and therefore the magnetic field, alternates with a known frequency of $16.67 \mathrm{~Hz}$ and has a changing amplitude. Even though the train used in the measurements had a Diesel engine, the periodic magnetic field was strongly pronounced in the measurements due to other electric trains in the vicinity.

In this paper, this issue is addressed with a band stop filter that damps this frequency in the measurements. Another, more severe problem is when the magnetic field changes due to changes in the environment. This can happen temporarily, e.g., when another train is passing by or permanently, e.g., due to construction work. This causes outliers in the measurements that have to be handled in the filter to avoid divergence. This issue will be discussed and addressed in the next section when the particle filter is introduced. More details on the magnetic field in the railway domain can be found in [8].

\section{IMPLEMENTATION}

\section{A. Topological Position}

In contrast to the positions of road vehicles, the positions of railway vehicles are limited to a track. Therefore, the train position can be described in relation to topology of the track network. The topological position of a train is uniquely described with the set

$$
\mathbf{p}=\{s, I\}
$$

where $s$ is the along-track position, describing the position on the current track, and $I \in \mathbb{N}_{0}$ is the ID of the current track. The along-track position is the distance the train has to drive from the start of the current track $I$ to its current position. The domain of $s$ on track $I$ therefore is $\left\{s \in \mathbb{R}^{+} \mid 0 \leq s \leq L_{I}\right\}$ where $L_{I}$ is the length of the track. The definition of $\mathbf{p}$ leads to discontinuities in $s$ whenever the borders of the track are reached. Handling these discontinuities requires the topology of the track network, that means the information how the tracks are interconnected to each other, and the ID of the current track. When a track changes at a switch, the position must be recalculated in respect to the new track and track ID. In the rest of the paper, the topology of the track network is assumed to be known. This assumption is reasonable, since the topology can only change during constructions and is then again constant. The topology information is easily obtained from different sources like OpenStreetMap or aerial pictures.

\section{B. Track Map}

For localization, the topology is stored in a track map. The map is a list of tracks, where each track contains the magnetic field vector measured at the different along-track positions, the corresponding ECEF position, and the information how the track is linked to the others. For the magnetic field, it is important to define how the magnetometer triad is oriented during the measurements of the map. In this paper, the $\mathrm{x}$ axis of the sensor frame is aligned with the direction of the track. More precisely, the magnetometer $\mathrm{x}$-axis is facing into driving direction of a train that drives from the end of a track at $s=L_{I}$ to the start of the track $s=0$. This definition is arbitrary, but must be maintained during the mapping to ensure that the magnetic field in the map is consistent in respect to the magnetometer orientation. In the particle filter, the map is 
considered to be a function that maps the topological position $\mathbf{p}$ to the magnetic field vector at that position

$$
\mathbf{m}: \mathbf{p} \mapsto \mathbf{m} \in \mathbb{R}^{3}
$$

\section{Joint Position Estimation and Track Identification}

1) State Space Model: This section is based on the particle filter from our prior work in [7]. The state space model of the particle filter is extended with an input from an accelerometer that is aligned with the $\mathrm{x}$-axis of the magnetometer. This assures that the sign of the acceleration measurements can be corrected with the train orientation which is estimated from the magnetometer measurements and the magnetic map of the track network. In addition, multiple observation models to cope with false measurements due to passing trains, outdated map information or noise from the overhead line are introduced to the filter. For the derivation of the particle filter, the trajectory, containing all track IDs the train traverses, is assumed to be known. Switches and therefore the identification of the correct new track ID and the discontinuities in the alongposition are handled in a second step based on the particle filter position estimate as will be described in the next sections. Since the track ID is known, the state vector $\mathbf{x}$ contains only the position $s$, the train speed $\dot{s}$, and the orientation of the accelerometer and the magnetometer $\mathcal{O}$

$$
\mathbf{x}=\left[\begin{array}{lll}
s & \dot{s} & \mathcal{O}
\end{array}\right]^{T} .
$$

The orientation is binary $\mathcal{O} \in\{-1,1\}$ and indicates if the magnetometer and accelerometer $\mathrm{x}$-axes are pointing towards the end $(\mathcal{O}=-1)$ or beginning $(\mathcal{O}=1)$ of the current track. The orientation ensures that the acceleration is integrated with the correct sign, which is also reflected in the discrete-time system model

$$
\mathbf{x}_{k}=\left[\begin{array}{lll}
1 & T & 0 \\
0 & 1 & 0 \\
0 & 0 & 1
\end{array}\right] \mathbf{x}_{k-1}+\left[\begin{array}{c}
0 \\
-\mathcal{O} T \\
0
\end{array}\right] a_{k-1}+\left[\begin{array}{l}
0 \\
1 \\
0
\end{array}\right] w_{k-1}
$$

with sampling time $T$, time step $k$, the measured acceleration $a_{k-1}$, and the accelerometer measurement noise $w_{k-1}$. The acceleration is multiplied with $-\mathcal{O}$ because of how the magnetometer was oriented during mapping and therefore how the orientation is defined. The orientation is assumed to be constant without any noise. So once the value is set it cannot change anymore. This was done because the orientation is fixed and usually does not change during operation. In the concrete particle filter implementation, half of the particles are initialized with $(\mathcal{O}=-1)$ and half with $(\mathcal{O}=1)$. After processing a few magnetic field measurements and resampling steps, only particles with the correct orientation remain. The bias of the accelerometer and the attitude of the train is not estimated because it was not necessary. The slope of an adhesion railway track is always close to zero, estimating the slope with an accuracy that is beneficial for the localization therefore requires gyroscopes with a very high quality. For the accelerometer bias, a fixed value was set at the initialization of the filter. If required, including the accelerometer bias into the state space model and the particle filter would be straightforward. With the definition of the state vector, the magnetometer measurement model can be described by

$$
\mathbf{z}_{k}=\operatorname{diag}\left(\left[\begin{array}{lll}
\mathcal{O} & \mathcal{O} & 1
\end{array}\right]\right) \cdot \mathbf{m}\left(s_{k}, I_{k}\right)+\mathbf{n}_{\mathbf{k}}
$$

where $\operatorname{diag}(\mathbf{v})$ is a diagonal matrix with $\mathbf{v}$ as its main diagonal entries and $\mathbf{n}_{\mathbf{k}}$ is the magnetometer measurement noise vector. In addition to the actual sensor noise, which is small and can be assumed Gaussian, the measurements are contaminated with periodic noise introduced by currents in the overhead line and distortions caused by passing trains or outdated values in the map. In particular, passing trains and changes in the magnetic field can cause issues in the filter because it is not easy to predict or to remove them by filtering in the frequency domain. Therefore multiple noise models, as proposed in [9], are used in the weight calculation of the filter. The main idea is to have a noise model for the nominal case when the map is up-to-date and the measurements are only contaminated with the sensor noise and a second model for all the error cases. The multiple noise models are integrated into a sampling importance resampling (SIR) particle filter as described e.g. in [10].

2) SIR Particle Filter: The SIR filter tries to estimate the posterior density of the state trajectory $\mathbf{x}_{0: k}$ from time step 0 to $k$ conditioned on the measurement history $\mathbf{z}_{0: k}$. The posterior is approximated with a set of $N_{p}$ weighted particles $\left\{\mathbf{x}_{0: k}^{i}, w_{k}^{i}\right\}$. The weighted particles can be interpreted as a Dirac mixture density that approximates the posterior

$$
p\left(\mathbf{x}_{0: k} \mid \mathbf{z}_{0: k}, a_{0: k-1}\right) \approx \sum_{i=1}^{N_{p}} w_{k}^{i} \delta_{\mathbf{x}_{0: k}^{i}}\left(\mathbf{x}_{0: k}\right)
$$

with the Dirac distribution $\delta_{\mathbf{x}_{0: k}^{i}}\left(\mathbf{x}_{0: k}\right)$ centered at the i-th particle position in the state space. The SIR particle filter processes the measurement data sequentially and utilizes a simple predict and update scheme. In each time step, first the prediction is performed by sampling, i.e., by drawing a new set of particle, from an importance density $q\left(\mathbf{x}_{k}^{i} \mid \mathbf{x}_{k-1}^{i}, \mathbf{z}_{k}, a_{k-1}\right)$ and second the weights of the predicted particles are adjusted with the equation

$$
\tilde{w}_{k}^{i}=w_{k-1}^{i} \frac{p\left(\mathbf{z}_{k} \mid \mathbf{x}_{k}^{i}\right) p\left(\mathbf{x}_{k}^{i} \mid \mathbf{x}_{k-1}^{i}, a_{k-1}\right)}{q\left(\mathbf{x}_{k}^{i} \mid \mathbf{x}_{k-1}^{i}, \mathbf{z}_{k}, a_{k-1}\right)} .
$$

Here, the importance density is set to the transition model $p\left(\mathbf{x}_{k}^{i} \mid \mathbf{x}_{k-1}^{i}, a_{k-1}\right)$ from (4) and therefore the weight update reduces to a multiplication of the weight from the previous time step with the likelihood $p\left(\mathbf{z}_{k} \mid \mathbf{x}_{k}^{i}\right)$ of the newest measurement. When all weights are updated, a renormalization is performed to ensure $\sum_{i} w_{k}^{i}=1$

$$
w_{k}^{i}=\frac{\tilde{w}_{k}^{i}}{\sum_{i=1}^{N_{p}} \tilde{w}_{k}^{i}}
$$

The particle filter is inherently unstable and degeneracy of the point cloud is unavoidable without appropriate counter measures. Degeneracy means that after a couple of update steps all but one particle have weights close to zero and therefore the point cloud is only a poor approximation of 
the true posterior. The problem is caused by a lack of a feedback mechanism that forces particles back to areas where the posterior is not close to zero. In the SIR filter, resampling therefore is performed whenever the particle weights become too unevenly distributed. A common measure for deciding when a resampling is necessary is the effective sampling size

$$
N_{\text {eff }}=\frac{1}{\sum_{i=1}^{N_{p}} w_{k}^{i^{2}}} \leq N_{p}
$$

If $N_{\text {eff }}$ falls below a defined threshold a new set of $N_{p}$ particles is drawn. The probability for each particle to be resampled is proportional to its weight.

\section{SIR with Multiple Noise Models}

The integration of multiple noise models follows the algorithm proposed in [9]. The idea is to calculate the posterior as the weighted sum of the posteriors resulting from the different noise models $M \in\left\{M_{j}\right\}_{j=1}^{N_{\text {Noise }}}$

$$
p\left(\mathbf{x}_{0: k} \mid \mathbf{z}_{0: k}, a_{0: k-1}\right)=\sum_{j=1}^{N_{\text {Noise }}} \mu_{k}^{j} \cdot p\left(\mathbf{x}_{0: k} \mid \mathbf{z}_{0: k}, a_{0: k-1}, M_{j}\right)
$$

with the probability $\mu_{k}^{j}$ that model $M_{j}$ is correct at time step $k$ given the measurements. Inserting the Dirac Mixture approximation (6) of the different noise models into (10) the posterior becomes

$$
\begin{aligned}
p\left(\mathbf{x}_{0: k} \mid \mathbf{z}_{0: k}, a_{0: k-1}\right) & =\sum_{j=1}^{N_{\text {Noise }}} \mu_{k}^{j} \sum_{i=1}^{N_{p}} w_{k}^{i, j} \delta_{\mathbf{x}_{0: k}^{i}}\left(\mathbf{x}_{0: k}\right) \\
& =\sum_{i=1}^{N_{p}} w_{k}^{i} \delta_{\mathbf{x}_{0: k}^{i}}\left(\mathbf{x}_{0: k}\right) .
\end{aligned}
$$

The weights $w_{k}^{i}$ of the averaged posterior are the weighted average of the weights $w_{k}^{i, j}$ of the different models

$$
w_{k}^{i}=\sum_{j=1}^{N_{\text {Noise }}} \mu_{k}^{j} w_{k}^{i, j} \text {. }
$$

The weights of the different models $w_{k}^{i, j}$ are derived based on the weight update (7), the model specific likelihood and the weight of the mixture from the previous step

$$
w_{k}^{i, j} \propto w_{k-1}^{i} p\left(\mathbf{z}_{k} \mid \mathbf{x}_{k}^{i}, M_{j}\right) .
$$

Note, in (11) the particles are the same for each component of the mixture. Only the weight calculation is carried out for each model, the prediction step is only performed once. In the next time step therefore particles can be considered as they have been drawn from the averaged posterior. The model probabilities from the previous time step $\mu_{k-1}$ are predicted with a simple model adopted from [9]

$$
\tilde{\mu}_{k}^{j}=\frac{\left(\mu_{k-1}^{j}\right)^{\alpha}}{\sum_{j=1}^{N_{\text {Noise }}}\left(\mu_{k-1}^{j}\right)^{\alpha}}
$$

with forgetting factor $\alpha \in(0,1)$. The predicted model probabilities are then updated with the following equation

$$
\mu_{k}^{j}=\frac{p\left(\mathbf{z}_{k} \mid \mathbf{z}_{0: k-1}, M_{j}\right) \tilde{\mu}_{k}^{j}}{\sum_{j=1}^{N_{\text {Noise }}} p\left(\mathbf{z}_{k} \mid \mathbf{z}_{0: k-1}, M_{j}\right) \tilde{\mu}_{k}^{j}} .
$$

The marginal model likelihood $p\left(\mathbf{z}_{k} \mid \mathbf{z}_{0: k-1}, M_{j}\right)$ is obtained from the marginalization over the state vector trajectory

$p\left(\mathbf{z}_{k} \mid \mathbf{z}_{0: k-1}, M_{j}\right)=\int p\left(\mathbf{z}_{k} \mid \mathbf{x}_{0: k}, M_{j}\right) p\left(\mathbf{x}_{0: k} \mid \mathbf{z}_{0: k}, a_{0: k-1}\right) d \mathbf{x}_{0: k}$.

Inserting (11) in (16) results in the approximation of the marginal likelihood

$$
p\left(\mathbf{z}_{k} \mid \mathbf{z}_{0: k-1}, M_{j}\right) \approx \sum_{i=1}^{N_{p}} w_{k}^{i} p\left(\mathbf{z}_{k} \mid \mathbf{x}_{0: k}^{i}, M_{j}\right) .
$$

\section{E. Point Estimate Calculation}

For localization, a point estimate of the train position must be derived from the posterior in (11). Here the estimate minimizing the Bayesian mean square error (MMSE) is chosen

$$
\begin{aligned}
\hat{\mathbf{x}}_{0: k} & =E\left[\mathbf{x}_{0: k} \mid \mathbf{z}_{0: k}, a_{0: k-1}\right] \\
& =\int \mathbf{x}_{0: k} p\left(\mathbf{x}_{0: k} \mid \mathbf{z}_{0: k}, a_{0: k-1}\right) d \mathbf{x}_{0: k},
\end{aligned}
$$

which can be approximated with the particle set

$$
\hat{\mathbf{x}}_{0: k} \approx \int \mathbf{x}_{0: k} \sum_{i=1}^{N_{p}} w_{k}^{i} \delta_{\mathbf{x}_{0: k}^{i}}\left(\mathbf{x}_{0: k}\right) d \mathbf{x}_{0: k}=\sum_{i=1}^{N_{p}} w_{k}^{i} \mathbf{x}_{0: k}^{i} .
$$

The MMSE therefore is the weighted sum of the particles.

\section{F. Track Identification}

Assuming the particle filter introduced above is working properly, the track identification procedure is straightforward. Every time when a particle is predicted over the starting point of an switch, a copy of the particle filter for each possible track hypothesis is created. Each track hypothesis therefore has its own filter that assumes the tracks to be known. On the basis of the MMSE position estimate of each filter and the magnetometer measurements, a test statistic is calculated. In particular, the likelihood ratio between two track hypothesis $\mathcal{H}_{0}=$ "left switchway" and $\mathcal{H}_{1}=$ "right switchway" is used

$$
\Lambda\left(\mathbf{z}_{k_{\text {split }}: k}\right)=\log \frac{p\left(\mathbf{z}_{k_{\text {split }}: k} \mid s_{k_{\text {split:k}}}^{\mathcal{H}_{0}}\right)}{p\left(\mathbf{z}_{k_{\text {split: }} \mid} \mid s_{k_{\text {split }}: k}^{\mathcal{H}_{1}}\right)} .
$$

The likelihood ratio considers all measurements starting with time step $k_{\text {split }}$ when the switch was first crossed. The measurements are assumed to be independent from each other, which enables recursive updates for $\Lambda$ every time a new measurement becomes available. In a likelihood ratio test the decision is made based on the inequality

$$
\Lambda\left(\mathbf{z}_{k_{\text {split }}: k}\right) \underset{\mathcal{H}_{1}}{\stackrel{\mathcal{H}_{0}}{\gtrless}} T
$$


with some threshold $T$ that depends on which metric is to be optimized, e.g., false alarm rate or number of missed detections [11]. Setting a theoretical threshold for the application considered in this paper is difficult, since the true distributions of the likelihoods are unknown. For simplicity, the likelihoods are assumed Gaussian and the threshold is set empirically. Also a dead band is added, the decision for one of the hypotheses therefore is made only if $\Lambda \leq-T$ or $\Lambda \geq T$ is fulfilled. This helps to avoid false decisions that were observed when the map has discontinuities at the border between two tracks. The discontinuities are caused by the mapping based on GNSS positions and are systematic errors in the map that are hard to avoid due to the limited accuracy of the positioning during mapping. Introducing a dead band solved this issue in a simple way and added robustness to the track identification. When there are more than two particle filter, e.g., when the train already passes the next switch before the last switch way is identified, a hypothesis is selected when its likelihood compared to all others is larger than the threshold.

\section{G. Joint Train Localization and Track Identification}

In this section, the particle filter for known tracks and the track identification are combined to a joint estimation algorithm. The joint algorithm extends each particle state (3) with the track ID $I$. In addition, for each particle filter the history $H$ of tracks that are traversed during the run are stored. The pseudo code of one iteration of the proposed joint estimator is shown in Algorithm 1. In the MMSE calculation of the along-track position in line 23 , an additional step is required that accounts for the case when not all particles are on the same track. In this case, an averaging over the along-track positions makes no sense, because the along-track position is discontinuous at the end and beginning of tracks. To enable averaging, first a virtual track is constructed by concatenating all tracks in the history. Secondly, the along-track position and the track ID of each particle is translated to an alongtrack position on the virtual track. All particle are now on the same track and the along-track position is continuously defined. After the MMSE is calculated, it is mapped back to a track ID $\hat{I}$ and an along-track position $\hat{s}$. This is the estimated topological position and the output of the filter.

\section{Measurement CAmpaign}

\section{A. Track network}

For the evaluation of the proposed algorithm ten runs between Augsburg main station and Friedberg station are considered. The same data was already used in our prior work [12] to evaluate the performance of a GNSS and IMU based localization approach and in [8] to analyze the suitability of the magnetic field for localization. The track network topology is shown in Fig. 2. The network contains six switches. Actually there are more switches in the main station at Augsburg, but only six switches are relevant. The rest had always been passed on the same switch way and therefore no measurements of the alternative switch ways are available. Overall, the network has a length of roughly $14.30 \mathrm{~km}$. Out of the ten runs, four were used to create the magnetic field map and six to evaluate the proposed localization algorithm. The trajectories through

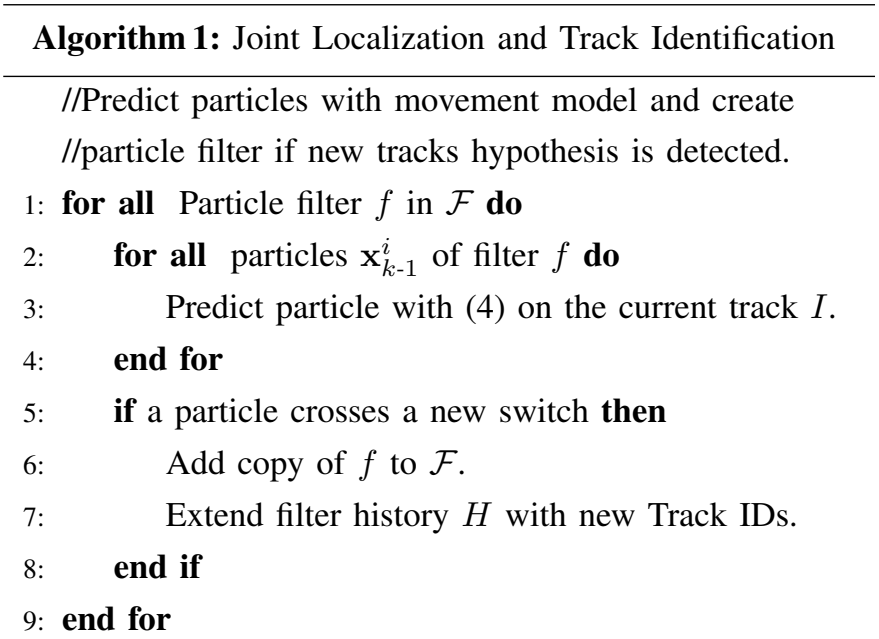

//Handle the discontinuities of the along-track position if //a particle moved on a new track and update the track ID

10: for all Particle filter $f$ in $\mathcal{F}$ do

for all particles $\mathbf{x}_{k}^{i}$ of filter $f$ do

if $\mathbf{x}_{k}^{i}$ crossed switch then

Set new track ID $I_{k}^{i} \in H$.

Set position $s_{k}^{i}$ on $I_{k}^{i}$.

end if

Get magnetic field $\tilde{\mathbf{z}}_{k}$ at position $s_{k}^{i}$ and $I_{k}^{i}$ (5).

Update model weights $w_{k}^{i, j}$ for all noise models

with $\tilde{\mathbf{z}}_{k}$, the measurement $\mathbf{z}_{k}$ and (13).

18:

end for

19: $\quad$ Predict model probabilities $\mu_{k-1}^{j}$ with (14).

20: $\quad$ Update and normalize $\mu_{k-1}^{j}$ with (15) and (17).

21: $\quad$ Normalize model weights $w_{k}^{i, j}$.

22: $\quad$ Update mixture weights $w_{k}^{i}$ 12.

23: Calculate MMSE position estimate $\hat{s}_{k}$ with (19) and the corresponding track ID $\hat{I}_{k}$.

24: $\quad$ Update the likelihood $p\left(\mathbf{z}_{k_{\text {split }}: k} \mid \hat{s}, \hat{I}_{k_{\text {split }}: k}\right)$ of $f$.

25: $\quad N_{\text {eff }}=1 / \sum_{i=1}^{N_{p}} w_{k}^{i^{2}}$.

26: $\quad$ if $N_{\text {eff }}<N_{\mathrm{T}}$ then

27: $\quad$ Resample and assign equal mixture weights.

28: $\quad$ end if

29: end for

30: Remove any filter from $\mathcal{F}$ if its likelihood ratio $\Lambda\left(\mathbf{z}_{k_{\text {split }}: k}\right)$ to another filter crosses threshold.

31: if Switch way is identified then

32: Output: Topological position $\hat{\mathbf{p}}=\{\hat{s}, \hat{I}\}$.

33: else

34: Output: Unique topological position does not exist. 5: end if 


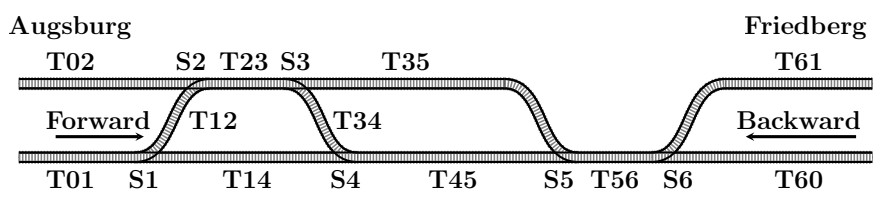

Fig. 2. Schematic drawing of the track network topology between Augsburg and Friedberg. All switches S1 - S6 and all tracks T1-T61 are passed multiple times during the measurements.

TABLE I

TRAJECTORIES OF THE DIFFERENT RUNS AND DRIVING DIRECTION

\begin{tabular}{ccc}
\hline Run & Trajectory & Direction \\
\hline 1 & T02, T23, T34, T45, T56, T60 & Forward \\
2 & T02, T23, T34, T45, T56, T60 & Forward \\
3 & T60, T56, T35, T23, T12, T01 & Backward \\
4 & T01, T14, T45, T56, T61 & Forward \\
5 & T60, T56, T35, T23, T02 & Backward \\
6 & T60, T56, T35, T23, T12, T01 & Backward \\
\hline
\end{tabular}

the network of the six runs for the evaluation are listed in Table I. Besides $S 4$, all switches are passed at least once from the direction from which it splits into two tracks and track identification became necessary. In total $S 1$ was passed once, $S 3$ twice and $S 2, S 5$ and $S 6$ were passed three times. The maximum speed during the measurements was $120 \mathrm{~km} / \mathrm{s}$.

\section{B. Hardware Setup}

The magnetic field and the acceleration was measured with an Xsens MTi at a rate of $200 \mathrm{~Hz}$. The sensor were build into a rack in the drivers cabin of an Alstom Coradia LINT 41. For mapping and getting the ground truth position and speed a u-blox LEA-6T low-cost single frequency receiver with a measurement rate of $1 \mathrm{~Hz}$ was used. Due to safety regulations, the GNSS antenna had to be mounted inside the train underneath a fiberglass roof.

\section{Map Creation}

To create the map, four of the ten runs were required to cover all possible tracks and switch ways. The positions of the switches were determined form aerial pictures and based on the switch positions and the GNSS positions the data sets were cut into tracks. For each track, the magnetic field measurement taken at a rate of $200 \mathrm{~Hz}$ was assigned to an along-track position $s$ by integrating the GNSS speed. This resulted in an unequally sampled map due the speed variations during measurements that was subsequently interpolated on a equidistant grid with a $10 \mathrm{~cm}$ spacing of the along-track position. Additionally to the magnetic field, the map also contains the ECEF positions to allow a comparison between the estimated topological position and the GNSS ground truth.

\section{Results}

The proposed algorithm was evaluated with the six runs from Table [ Each run lasted between $10 \mathrm{~min}$ and $15 \mathrm{~min}$ in which a distance of $\approx 7 \mathrm{~km}$ was traveled. The evaluation is split into two parts. First, the position accuracy is evaluated
TABLE II

POSITION AND SPEED RMSE

\begin{tabular}{ccc}
\hline & \multicolumn{2}{c}{ RMSE } \\
Run & Position [m] Speed [m/s] \\
\hline 1 & 4.46 & 0.28 \\
2 & 3.99 & 0.31 \\
3 & 5.03 & 0.22 \\
4 & 5.17 & 0.21 \\
5 & 6.45 & 0.22 \\
6 & 4.10 & 0.19 \\
Overall & 4.90 & 0.23 \\
\hline
\end{tabular}

and second, the result of the track identification is analyzed. For each data set, 100 Monte Carlo runs of the particle filter were performed to show that it works consistently. For the evaluation, we simply picked the result of the Monte Carlo run with the highest RMSE because the average accuracy was close for all runs.

During the evaluation, 3000 particles are used and the filter is updated with $10 \mathrm{~Hz}$. With these parameters the filter, implemented in MATLAB, was running in real time. Theoretically, the different particle filters could also run in parallel if higher update rates are required. To handle outliers, two noise models were used. The first model is Gaussian $\mathcal{N}\left(\mathbf{0}, \boldsymbol{\Sigma}_{\text {nom }}\right)$ for the nominal case with an covariance matrix $\boldsymbol{\Sigma}_{\text {nom }}$ of $1.3 \times 10^{-3} I_{3 \times 3}$ and the second model is a multivariate tdistribution $t\left(0, \boldsymbol{\Sigma}_{\mathrm{err}}, \nu\right)$ for the error case. The t-distribution has one degree of freedom $\nu=1$ and is much wider with $\boldsymbol{\Sigma}_{\mathrm{err}}=4.5 \times 10^{-2} I_{3 \times 3}$.

\section{A. Position and Speed Accuracy}

The position and speed accuracy shown in this section is calculated from the results of the particle filters that were following the correct track hypothesis. Showing the result for the particle filters on the wrong track is neglected here because they only existed for a few seconds, until they were deleted and the correct track was identified. In addition the position estimate on the wrong track has no meaning, since it is always wrong due to the wrong track assumption.

The position error is calculated from the GNSS ECEF position $\mathbf{p}_{\mathrm{ECEF}}$ and the ECEF position $\hat{\mathbf{p}}_{\mathrm{ECEF}}$ stored in the track map at the current MMSE estimate of the topological train position $\hat{\mathbf{p}}=\{\hat{s}, \hat{I}\}$

$$
\boldsymbol{\epsilon}_{\mathrm{pos}}=\mathbf{p}_{\mathrm{ECEF}}-\hat{\mathbf{p}}_{\mathrm{ECEF}}=\left[\begin{array}{lll}
\epsilon_{x} & \epsilon_{y} & \epsilon_{z}
\end{array}\right]^{T} .
$$

The root mean square error (RMSE) is

$$
\mathrm{RMSE}_{\mathrm{pos}}=\sqrt{\frac{1}{K} \sum_{k=1}^{K} \boldsymbol{\epsilon}_{k, \mathrm{pos}}^{T} \boldsymbol{\epsilon}_{k, \mathrm{pos}}}
$$

where $K$ is the number of measurements. For the speed error $\epsilon_{\text {speed }}$, simply the GNSS speed $\dot{s}_{\text {GNSS }}$ is subtracted from the MMSE speed estimate of the particle filter. The resulting RMSE value for position and speed is shown in Table II In Fig. 3 and Fig. 4, in addition the cumulative distribution 


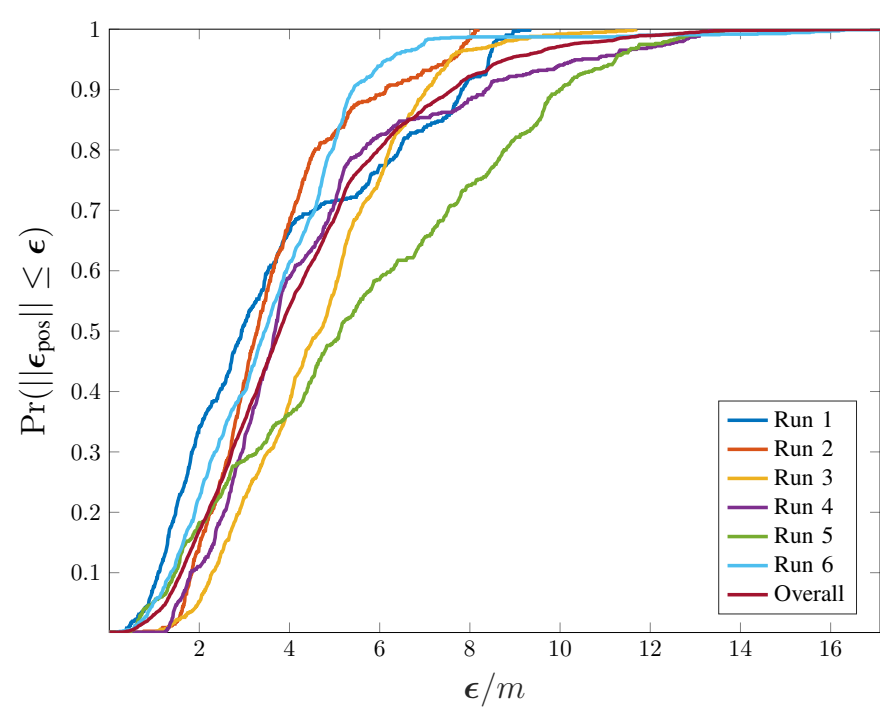

Fig. 3. CDF of the absolute position error.

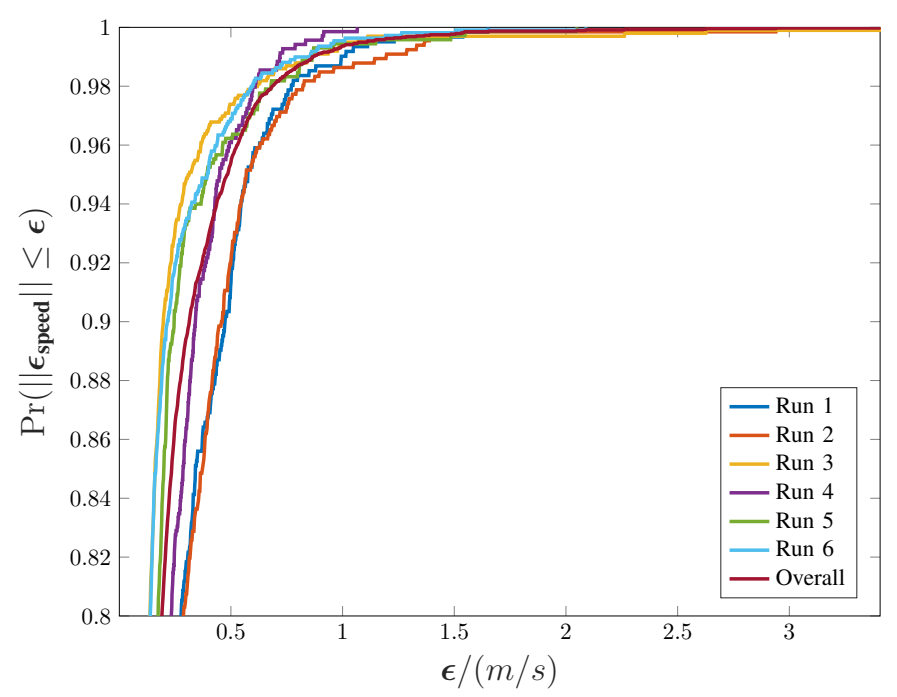

Fig. 4. CDF of the absolute speed error.

function (cdf) of the absolute errors is shown. The RMSE for the different runs are close to each other and below $7 \mathrm{~m}$. The overall RMSE calculated with the errors of all six runs is below $5 \mathrm{~m}$. Considering that the GNSS ground truth also contains errors in this order of magnitude, the positing accuracy looks promising. Here it should be noted that the map of track 45 had a systematic error that was most likely caused by a train passing by during the measurements for mapping. Nevertheless, the filter was able to estimate the position also for the runs which are driving over track 45, proving the robustness of the filter against outdated and therefore false map data. The same holds for the speed estimation. The speed was estimated with an high accuracy throughout the whole evaluation. The larger errors in Fig. 4 are partially caused by the GNSS ground truth, which had sporadic speed errors up to $2.5 \mathrm{~m} / \mathrm{s}$.
TABLE III

TIME DISTANCE UNTIL SWITCH WAY IS DETECTED

\begin{tabular}{ccccccccc}
\hline Switch & & Run 1 & Run 2 & Run 3 & Run 4 & Run 5 & Run 6 \\
\hline \multirow{2}{*}{ S1 } & Time / s & - & - & - & 4.22 & - & - \\
& Dist. / m & - & - & - & 67.00 & - & - \\
\multirow{5}{*}{ S2 } & Time / s & - & - & 6.20 & - & 6.60 & 4.59 \\
& Dist. / m & - & - & 37.14 & - & 36.54 & 26.28 \\
S3 & Time / s & 2.62 & 2.71 & - & - & - & - \\
& Dist. / m & 37.72 & 41.08 & - & - & - & - \\
S4 & Time / s & - & - & - & - & - & - \\
& Dist. / m & - & - & - & - & - & - \\
\multirow{2}{*}{ S5 } & Time / s & - & - & 2.61 & - & 2.60 & 2.61 \\
& Dist. / m & - & - & 39.26 & - & 39.88 & 39.81 \\
\multirow{2}{*}{ S6 } & Time / s & 6.19 & 6.48 & - & 2.76 & - & - \\
& Dist. / m & 111.64 & 103.76 & - & 41.74 & - & - \\
\hline
\end{tabular}

\section{B. Track Identification}

The track identification was able to detect the correct switch way for all runs. In Table III the duration and distances required to decide for the correct switch way is shown. The values in the table are the average over all Monte Carlo runs. The switch detection requires a few seconds and above $\approx 26 \mathrm{~m}$ to decide for the correct track. Before that the magnetic field for both tracks seems to be close to each other, which leads only to small differences between the likelihoods of the different hypothesis. A bigger spread in the detection times was observed for S2 and switch S6. For switch S6 the time is more than doubled. A look at the trajectories in Table 1 shows that the train was taking the same way in run 1 and run 2 and a different route for run 4 , what could explain the difference. But for S2 this effect is not observed, here the time is similar for run 3 and 5 which have different switch ways. Therefore and due to the small number of passes over each switch, no clear pattern could be found.

\section{CONCLUSIONS}

In this paper, we proposed a method that enables the joint estimation of the along-track position and track ID in real time, resulting in an estimate of the topological position. The method requires only a triad of magnetometers mounted inside the train, a single accelerometer pointing into the driving direction, and a map of the magnetic field along the railway track. The particle filter used for along-track localization was extended to contain multiple noise models, which introduced robustness against outliers caused by outdated map information, passing trains, and periodic noise form the overhead line. The track identification relies on multiple copies of the particle filter. Each copy of the filter follows another track hypothesis. With the estimated positions of the different filters and the magnetometer measurements, the likelihood ratio of the different hypotheses was calculated. A decision for one track is made when the ratio crosses a threshold. In a evaluation with over $60 \mathrm{~min}$ of data, measured on a $14.30 \mathrm{~km}$ long track network, it was shown that the correct track could be successfully identified in all cases. The identification required 
only a few seconds in which the train traveled approximately between $26 \mathrm{~m}-112 \mathrm{~m}$. The along-track localization showed also promising results and was able to continuously track the position even in the presence of measurement errors. The overall RMSE was below five meters and therefore close to the expected value from the single frequency GNSS receiver used as ground truth.

\section{ACKNOWLEDGMENT}

We want to thank the railway transportation company BRB ("Bayerische Regiobahn") for their cooperation with the measurement recordings.

\section{REFERENCES}

[1] J. Marais, J. Beugin, and M Berbineau, "A survey of GNSS-based research and developments for the European railway signaling," IEEE Transactions on Intelligent Transportation Systems, vol. PP, no. 99, pp $1-17,2017$.

[2] J. Haverinen and A. Kemppainen, "Global indoor self-localization based on the ambient magnetic field," Robotics and Autonomous Systems, vol. 57, no. 10, pp. 1028-1035, Oct. 2009. [Online]. Available: http://www.sciencedirect.com/science/article/pii/S0921889009001092

[3] M. Kok and A. Solin, "Scalable Magnetic Field SLAM in 3D Using Gaussian Process Maps," in 2018 21st International Conference on Information Fusion (FUSION), Jul. 2018, pp. 1353-1360.

[4] M. Frassl, M. Angermann, M. Lichtenstern, P. Robertson, B. J. Julian, and M. Doniec, "Magnetic maps of indoor environments for precise localization of legged and non-legged locomotion," in 2013 IEEE/RS International Conference on Intelligent Robots and Systems, Nov. 2013, pp. 913-920.
[5] J. A. Shockley and J. F. Raquet, "Navigation of ground vehicles using magnetic field variations," Navigation, vol. 61, no. 4, pp. 237-252, 2014 , navi.70.

[6] A. Canciani and J. Raquet, "Airborne Magnetic Anomaly Navigation," IEEE Transactions on Aerospace and Electronic Systems, vol. 53, no. 1, pp. 67-80, Feb. 2017.

[7] B. Siebler, O. Heirich, and S. Sand, "Train Localization with Particle Filter and Magnetic Field Measurements," in 2018 21st International Conference on Information Fusion (FUSION), Jul. 2018, pp. 1-5.

[8] O. Heirich, B. Siebler, and E. Hedberg, "Study of Train-Side Passive Magnetic Measurements with Applications to Train Localization," 2017. [Online]. Available: https://www.hindawi.com/journals/js/2017/ 8073982/abs/

[9] B. Liu, "Robust particle filter by dynamic averaging of multiple noise models," in 2017 IEEE International Conference on Acoustics, Speech and Signal Processing (ICASSP), Mar. 2017, pp. 4034-4038, iSSN: 2379-190X.

[10] M. S. Arulampalam, S. Maskell, N. Gordon, and T. Clapp, "A tutorial on particle filters for online nonlinear/non-Gaussian Bayesian tracking,' IEEE Transactions on Signal Processing, vol. 50, no. 2, pp. 174-188, Feb. 2002.

[11] H. L. Van Trees, Detection, estimation, and modulation theory, part I: detection, estimation, and linear modulation theory. John Wiley \& Sons, 2004.

[12] O. Heirich, "Bayesian Train Localization with Particle Filter, Loosely Coupled GNSS, IMU, and a Track Map," 2016. [Online]. Available: https://www.hindawi.com/journals/js/2016/2672640/ 